\title{
DYNAMIC CALIBRATION OF INDUSTRIAL ROBOTS WITH INERTIAL MEASUREMENT SYSTEMS
}

\author{
T. Alban, H. Janocha \\ Laboratory for Process Automation (LPA), \\ University of Saarland, Im Stadtwald, Geb. 13, \\ D-66123 Saarbrücken, Germany \\ fax : +49-681-302-2678, e-mail : alban@lpa.uni-sb.de
}

Keywords : Dynamic calibration, robot calibration, path accuracy, inertial navigation.

\begin{abstract}
Industrial robots require a high path accuracy for demanding continuous path-control tasks. In this publication we will present a new method which improves the path accuracy of industrial robots according to ISO 9283. This dynamic calibration is realized by means of a model based precontrol and corrects on-line the desired joint positions. For the identification of the model parameters of the precontrol the robot's path deviations are directly measured off-line at the end effector with an inertial measurement system. The experimental results presented here prove the efficiency of this dynamic calibration procedure.
\end{abstract}

\section{Introduction}

Control tasks for six degree-of-freedom robots in which the end effector has to follow any kind of trajectory as fast and precisely as possible require high standards of path accuracy. This is particularly true when off-line programming methods are applied. This has led to much research and development work aimed at a robot measurement with external measurement systems $[1,2,3]$ and an accuracy improvement of the industrial robots $[4,5,6]$. In this context most of the principles and procedures known aspire to improve static parameters according to ISO 9283 [7], such as for example pose accuracy or pose repeatability. With increasing speed the robot behaviour is determined by time dependent interaction between inertial, centrifugal, gravitational as well as Coriolis forces and torques on the one hand and forces and torques working at the driving end on the other hand. These and other conditions (elasticity, joint backlash) must be taken into account by means of dynamic calibration. In this context some methods have been proposed in which the robot internal sensors are used for measuring $[8,9]$. Therefore not all the causes for path deviation are taken into account.

In this publication a new method for dynamic robot calibration is presented that is based on the dynamic measuring of the end effector pose with an external inertial measurement system. The calibration is based on firstly modelling the error-causing conditions in analogy with the static robot calibration and then identifying the parameters of the precontrol by means of the path errors measured off-line and finally, with this in mind, performing the on-line correction of the desired values aiming to improve accuracy.

\section{Measuring of the robot's errors}

If dynamic robot errors, such as path deviation, are measured with the robot's internal sensors, not all relevant parameters can be recorded. For instance, the robot's internal sensors often measure the joint angle position on the driving end which is then used to calculate the joint angles by means of the drives transmission ratio; consequently gear errors cannot be recorded by the robot's internal sensors. That is why in this calibration procedure the robot's poses are measured with an inertial measurement system mounted to the end effector. This procedure makes it possible to record all sources of errors in the kinematic chain.

The inertial measurement system consists of three servo accelerometers and three ring laser gyros [3]. The principle of the inertial measurement system is shown in Fig. 1. After the measured data (acceleration and rotation rate) have undergone a error correction and the earth's rate and the earth gravitation have been subtracted, translation and rotation of this measurement system are given in an earth-fixed reference coordinate system (W). However, as the robot's joint values are required for the parameter identification in the joint space the recorded values have to be converted to the robot's base coordinates (R). For this, the relations of the different coordinate systems, presented by ${ }^{\mathrm{R}} \underline{\underline{T}}_{\mathrm{W}}$ and ${ }^{\mathrm{S}} \underline{\underline{T}}_{\mathrm{H}}$, have to be identified in a wrist/sensor calibration procedure that has been de- 


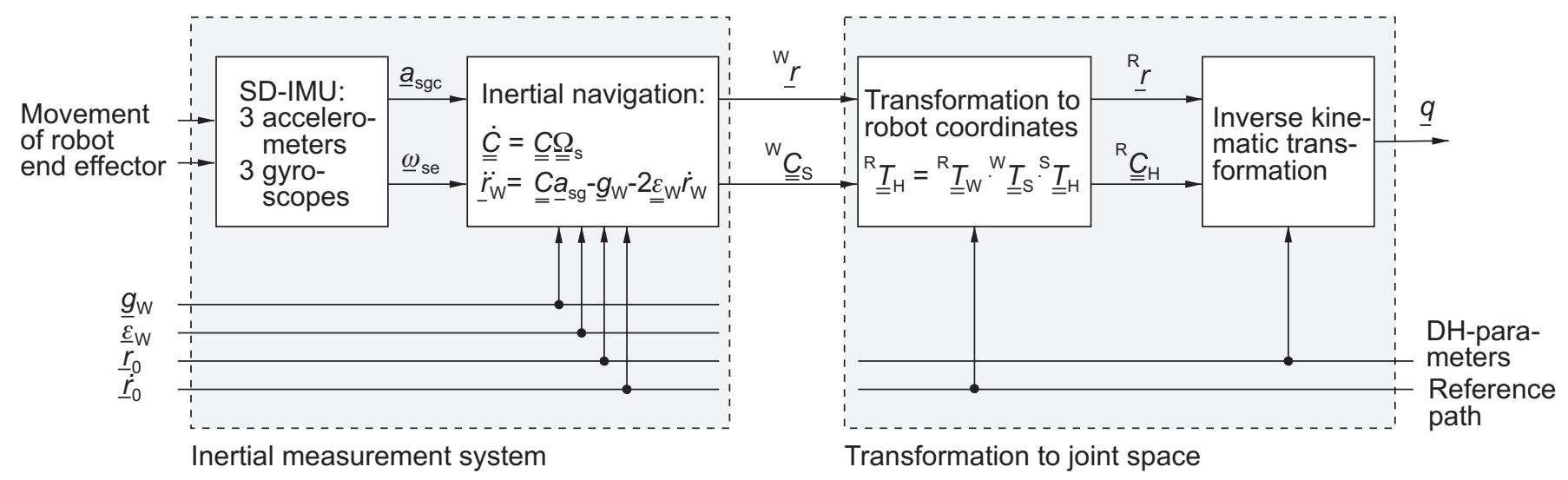

Fig. 1: Principle of an inertial measurement system

veloped for this purpose. Finally the values have to be transformed to the joint space of the robot by the inverse kinematic transformation.

Fig. 2 shows the measured joint angle errors $\Delta \underline{q}=\underline{q}_{\mathrm{d}}-\underline{q}$ of a typical industrial robot. The desired path was programmed as a straight line with a length of $800 \mathrm{~mm}$, to be executed at a speed of $0.4 \mathrm{~m} / \mathrm{s}$. The errors were recorded with the robot's internal sensors and with an inertial measurement system. In spite of differences caused mainly by
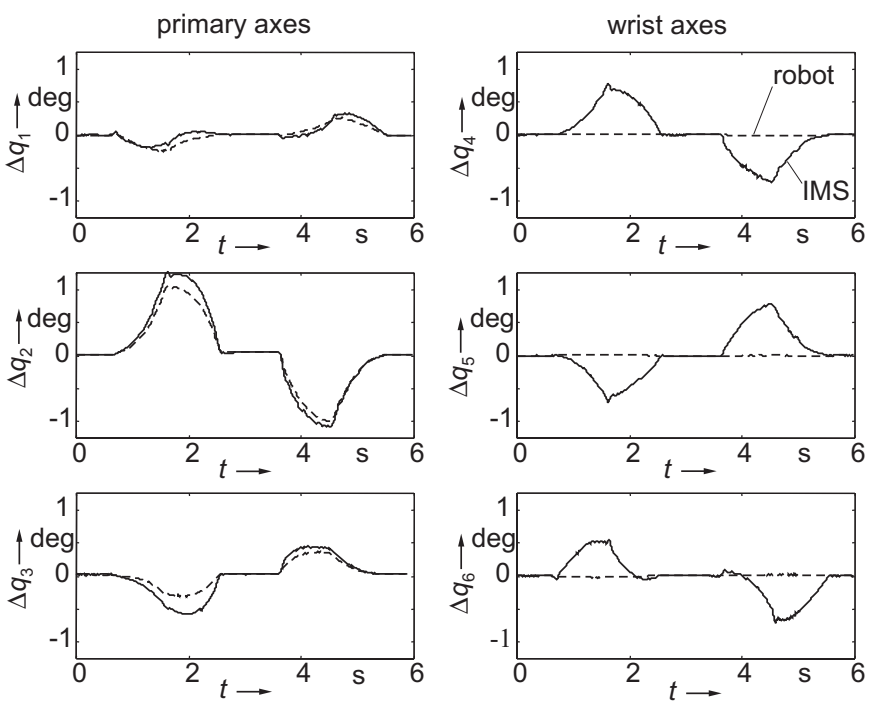

Fig. 2: Joint angle errors. Measured with robot's internal sensors and with an inertial measurement system (IMS)

compliance of the gears the errors of the primary axes measured with internal sensors are comparable to the errors measured with the external measurement system at the end effector. But the errors of the wrist are barely recorded by the internal sensors, because the gears of the wrist have a complex structure and their compliance only can be detected at the end of the kinematic chain.

\section{Dynamic robot calibration}

\subsection{Overview}

ISO 9283 describes criteria for the evaluation of the accuracy of industrial robots. For control tasks that have to take a given pose as precisely as possible, for example in spot welding, in particular the static parameters

- absolute accuracy or pose accuracy and

- pose repeatability

of the robot are important. Here the absolute accuracy indicates the precision with which the robot can take a programmed pose. The pose repeatability indicates the uncertainty with which the attained poses of the robot diverge from each other when the same desired pose is taken repeatedly by the robot.

Another parameter described in ISO 9283 is the

- path accuracy.

It indicates the deviation of robot end effector from the desired path while following a given contour. This characteristic is important e.g. for laser manufacturing operations in the field of welding and cutting of three-dimensional plate products. In the automobile industry the requirements for contour accuracy of plate products lie below one millimetre at a manufacturing velocity of several metres per minute. Such high requirements for accuracy can be well met with gantry robots whereas industrial robots with rotatory joints can show path deviations of several millimetres already at lower velocities. There are two reasons why this dynamic parameter according to ISO 9283 was not regarded as very important in the past. On the one hand there were no measuring systems available that could measure a robot path in a dynamic and practical way. On the other hand for the most tasks the path accuracy of the robot was of minor importance compared to the static pose accuracy. As the path accuracy of industrial robots is continuously increasing in importance 
in modern applications, and as in the meantime there are suitable measuring systems for the determination of the path deviation, the dynamic parameters of a robot become more and more important.

The most important causes for path deviations of industrial robots are listed in Table $1[10,11]$. As the static pose deviations also lead to path deviations, their causes are listed in the same table. The causes for errors which exclusively determine path accuracy and have no influence on pose accuracy are written in italics.

\begin{tabular}{|l|l|l|}
\hline Mechanics & Kinematics & Dynamics \\
\hline gear errors: & link length & dynamic of the \\
backlash, & deviation & real system \\
compliance & displacement of & following error \\
static, dynamic & axes & $\begin{array}{l}\text { simplified joint } \\
\text { and thermal }\end{array}$ \\
deformation & zero error & \\
angle transmis- \\
sion error
\end{tabular}

Table 1: Causes for path deviation of industrial robots

Static pose deviations can be modelled with the help of known procedures for static robot calibration and can be taken into account when programming the robot $[10,6$, $12,4,13,14]$. But the dynamic motion behaviour is not influenced by static calibration and has to be improved by other means.

The robot dynamics can be modelled by the differential equation (1). This dynamic model describes the forces and torques which act on the single joints and which are determined by the position, the velocity and the acceleration of the axes as well as by the forces and torques acting on the end effector.

$$
\underline{\tau}_{\mathrm{A}}=\underline{\underline{M}}(\underline{q}) \cdot \underline{\ddot{q}}+\underline{h}(\underline{q}, \underline{\dot{q}})+\underline{g}(\underline{q})+\underline{\tau}_{\mathrm{R}}(\underline{q}, \underline{\dot{q}})
$$

where

$\underline{q}, \dot{q}, \underline{q}: \quad(\mathrm{n} \times 1)$-vector of joint coordinates

$\underline{\tau}_{\mathrm{A}}: \quad(\mathrm{n} \times 1)$-vector of driving forces

$\underline{M}(q): \quad(\mathrm{n} \times \mathrm{n})$-inertia matrix

$\underline{\underline{h}(q}, \underline{q}): \quad(\mathrm{n} \times 1)$-vector of centripetal and Coriolis forces

$\underline{g}(\underline{q}): \quad(\mathrm{n} \times 1)$-vector of gravitational forces

$\underline{\tau}_{\mathrm{R}}(\underline{q}, \underline{\dot{q}}): \quad(\mathrm{n} \times 1)$-vector of frictional forces

The dynamic model is the basis of a controller design. Thus, the path accuracy depends on the quality of the model description, the parameter identification and the joint controller based on this. In this description of the dynamic behaviour the robot is assumed to be a rigid multi-body system with ideal joints without backlash. In a first approximation this is allowed because of the high transmission ratio of the gears used in robot axis, but at high path velocities or when drive backlash occurs, this leads to errors. Also the simplified structure of the joint controllers often used in industrial robots contributes to a reduction in path accuracy.

\subsection{Principle of the dynamic robot cali- bration}

The path deviation of a robot is the addition of the absolute pose deviation that does not depend on the velocity of the procedure and of the influences originated by dynamics (see Table 1). For this reason also a static robot calibration contributes to an improvement of path accuracy, but not to the same extent as a dynamic calibration that improves the robot's dynamic behaviour.

The procedure for the implementation of a dynamic robot calibration includes the following steps in analogy to the static robot calibration:

1. modelling,

2. measuring of the robot's errors and identification of the parameters,

3. error compensation, and

4. verification.

Fig. 3 provides an overview of the principle of the developed dynamic calibration procedure. For the improvement of the dynamic behaviour a component "error compensation" is added to the industrial robot; this component estimates the expected error of the joint angle $\Delta \underline{q}$ and transfers it to the robot control as an offset for the desired joint value. Today many manufacturers of robots offer an interface in the control unit that enables the correction of the desired values. The parameters of the model-based error compensation are identified through an external measurement with an inertial measurement system that has to be performed only once at the time of the calibration procedure.

\subsection{Model of the robot errors}

The model for the estimation of the joint angle errors from the desired joint angle values provided by the robot control has to describe linear as well as nonlinear effects and should be valid for the whole of the robot's working envelope. A complete physical description of the robot dynamics is too complex for an error compensation to be performed on-line. The motion behaviour is not only determined by elasticities and cross couplings but also by the structure of the unknown joint controller. Furthermore the error compensation must fulfil real-time requirements.

For this reason, the joint angle errors are described separately in simple, linear and nonlinear models whose parameters depend on input variables such as joint position, payload, velocity, etc. 


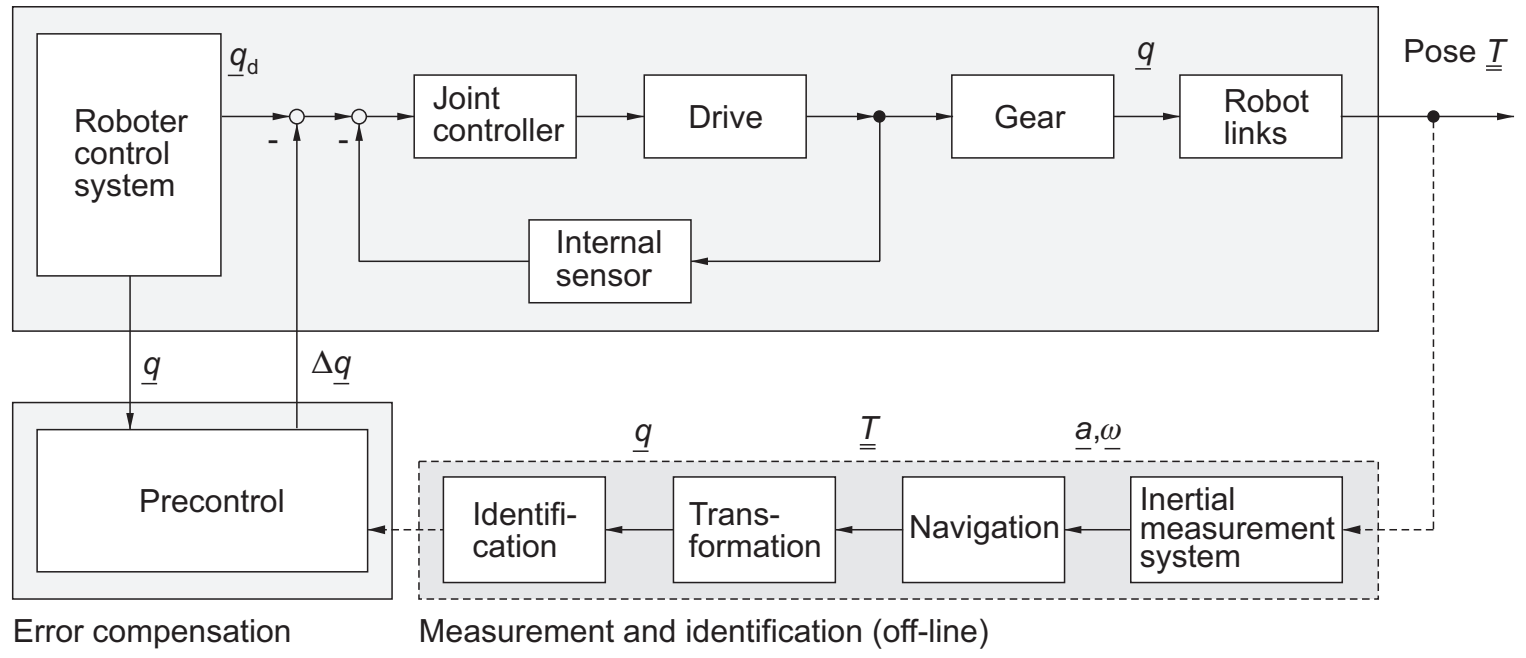

Fig. 3: Structure of the dynamic robot calibration

Thus, the angle errors for the robot joint i can be estimated by the following discrete linear model.

$$
\begin{gathered}
\hat{q}_{\mathrm{i}}(k T)=-a_{\mathrm{i}, 1}^{\star} \hat{q}_{\mathrm{i}}((k-1) T)-\ldots \\
\left.+b_{\mathrm{i}, 0}^{\star} q_{\mathrm{d}, \mathrm{i}}(k-d) T\right)+b_{\mathrm{i}, 1}^{\star} q_{\mathrm{d}, \mathrm{i}}((k-d-1) T)+\ldots \\
\Delta \hat{q}_{\mathrm{i}}(k T)=q_{\mathrm{d}, \mathrm{i}}((k-d) T)-\hat{q}_{\mathrm{i}}(k T)
\end{gathered}
$$

where

i: $\quad$ joint $(\mathrm{i}=1 \ldots 6)$

$q_{\mathrm{d}, \mathrm{i}}: \quad$ desired value for the joint $\mathrm{i}$

$d: \quad$ dead time

$a_{\mathrm{i}, \mathrm{j}}^{\star}$ : $\quad$ parameter $a$ depending on boundary conditions

$b_{\mathrm{i}, \mathrm{j}}^{\star}$ : $\quad$ parameter $b$ depending on boundary conditions

The experimental research conducted so far has shown that considering payload and joint angle velocity brings advantages as far as improvement in precision is concerned. Also if the robot operates at the edge of its working envelope, the values of the identified parameters change. In the model of error compensation the marginal conditions are taken into consideration through the parameters $a$ and $b$ that depend on the operating points. For this purpose the parameters are stored in a multidimensional table that is addressed depending on the operating point. In the next example of a reduced parameter table the assumption is made that the model parameters depend on the joint angle velocity $\dot{q}$ and on the payload $m$. When indicating the parameters the operating point is marked with two superior indices. Thus, for the determination of the model parameters results Table 2 which is addressed through the joint angle velocity and the payload applied to the end effector.

The procedure can be extended to several dimensions in order to take also into account influence factors such as

\begin{tabular}{c|c|c|}
\multirow{1}{*}{$\dot{q}: 0 \ldots 0.2 \dot{q}_{\max }$} & $m: 0 \ldots 0.5 m_{\mathrm{N}}$ & $m: 0.5 \ldots 1 m_{\mathrm{N}}$ \\
\cline { 2 - 3 }$\dot{q}: 0.2 \ldots 0.8 \dot{q}_{\max }$ & $\underline{a}_{\mathrm{i}}^{0,0}, \underline{b}_{\mathrm{i}}^{0,0}$ & $\underline{a}_{\mathrm{i}}^{0,1}, \underline{b}_{\mathrm{i}}^{0,1}$ \\
\cline { 2 - 3 }$\dot{q}: 0.8 \ldots 1 \dot{q}_{\max }$ & $\underline{a}_{\mathrm{i}}^{1,0}, \underline{b}_{\mathrm{i}}^{1,0}$ & $\underline{a}_{\mathrm{i}}^{1,1}, \underline{b}_{i}^{1,1}$ \\
\cline { 2 - 3 } & $\underline{a}_{\mathrm{i}}^{2,0}, \underline{b}_{\mathrm{i}}^{2,0}$ & $\underline{a}_{\mathrm{i}}^{2,1}, \underline{b}_{\mathrm{i}}^{2,1}$ \\
\hline
\end{tabular}

where

i: $\quad$ joint $(\mathrm{i}=1 \ldots 6)$

$\dot{q}, \dot{q}_{\max }: \quad$ actual and maximum velocity of joint $\mathrm{i}$

$m, m_{\mathrm{N}}$ : payload and nominal load

$\underline{a}_{i}^{\mathrm{k}, 1}, \underline{b}_{i}^{\mathrm{k}, 1}: \quad$ vectors of parameters depending on boundary conditions

Table 2: Example for calculating the variable model parameters

the position of the axes.

Nonlinear effects such as backlash and the robot transient compensation behaviour which influences the pose stabilization time will be modelled through characteristic transfer elements in a following step. The joint angle error caused by backlash can be modelled by the following two-point characteristic line.

$$
\Delta \hat{q}_{\mathrm{i}}^{\mathrm{bl}}(k \Delta t)=\left\{\begin{array}{rll}
l_{\mathrm{i}} & , & \dot{q}_{\mathrm{i}}>0 \\
-l_{\mathrm{i}} & , & \dot{q}_{\mathrm{i}}<0
\end{array}\right.
$$

where

i: $\quad$ joint $(i=1 \ldots 6)$

$\hat{q}_{\mathrm{i}}^{\mathrm{bl}}$ : $\quad$ angle deviation of joint $\mathrm{i}$ caused by backlash

$\dot{q}_{\mathrm{i}}$ : $\quad$ angle error of joint $\mathrm{i}$

$l_{\mathrm{i}} \quad$ backlash of joint $\mathrm{i}$ 


\subsection{Implementation of the precontrol}

The error compensation has been implemented for a typical industrial robot and is based on the robot's interface offered by the manufacturer for adapting the control parameters and for adding offsets to the given desired values. The structure of the compensation element is shown in Fig. 4.

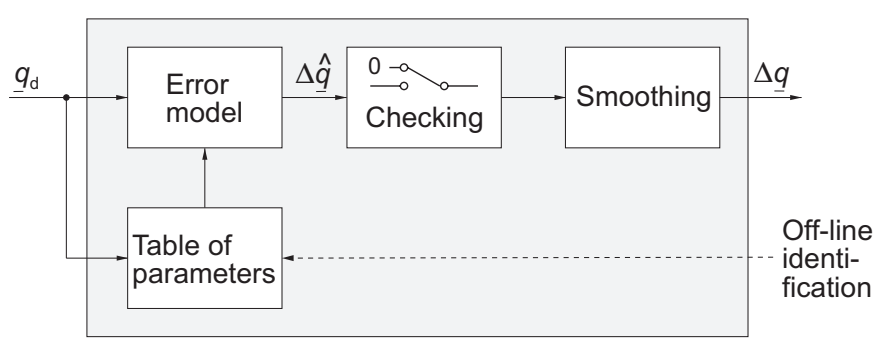

Fig. 4: Structure of the error compensation

In the current configuration the error compensation that is performed on-line is based on a linear error model in accordance with equation 2 and calculates the joint angle error $\Delta \underline{q}$ on the basis of the joint angle ideal values $q_{\mathrm{d}}$ provided by the robot control. As stability tests cannot be carried out because of the in general unknown behaviour of the modified plant, the error compensation contains a control unit. It checks the estimated angle errors that are transferred to the robot control as joint angle offsets. If a given tolerance is exceeded, the error compensation is deactivated. Then the calculated correction values are smoothened; with this method continuous joint angle offsets can be achieved.

\section{Experimental results}

For verifying the dynamic robot calibration a discrete model has been installed for the main joints of the industrial robot under test (see equation 2). The optimal order of the model was $n=32$ for the sample time of 2 ms which corresponds to one cycle of the attitude control of the robot joint controllers. The identification of the parameters has been carried out with a linear estimating technique on the basis of the joint angles measured with the inertial measurement system. For the measurement the single robot joints were moved at the highest possible acceleration.

The path programmed for the test was a straight line at the outer limits of the working envelope. It was followed by the robot in both directions with a maximal path velocity of $v=0.4 \mathrm{~m} / \mathrm{s}$ and a payload of $5 \mathrm{~kg}$ (corresponding to $1 / 3$ of the nominal payload). The end effector should with continuous orientation - follow the desired path with an extension of $s=800 \mathrm{~mm}$ in negative $y$-direction and then after a pause of $1 \mathrm{~s}$ come back to the starting point following the same line. First the not calibrated robot and then the calibrated one moved along this path under the same conditions. Fig. 5 shows a three-dimensional view of the desired path of the robot end effector and of the actual paths measured with the inertial measurement system and performed by the calibrated and by the not calibrated robot.

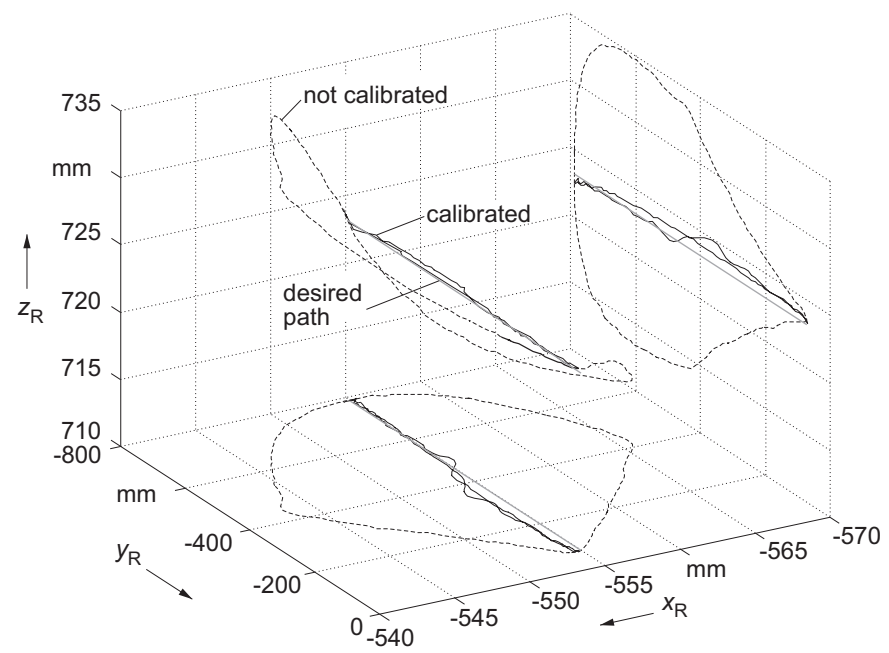

Fig. 5: Measured robot path

For reasons of clearness the paths are projected to the $x y$-plane and to the $y z$-plane. The large path deviations of the actual path of the not calibrated robot are due to the position of the test path at the outer limits of the working envelope and to the relatively high path acceleration.

For a better evaluation of the path accuracy, the path deviation calculated according to ISO 9283 on the basis of the perpendicular dropped from the actual path to the desired path is shown in Fig. 6. The maximal path devi-
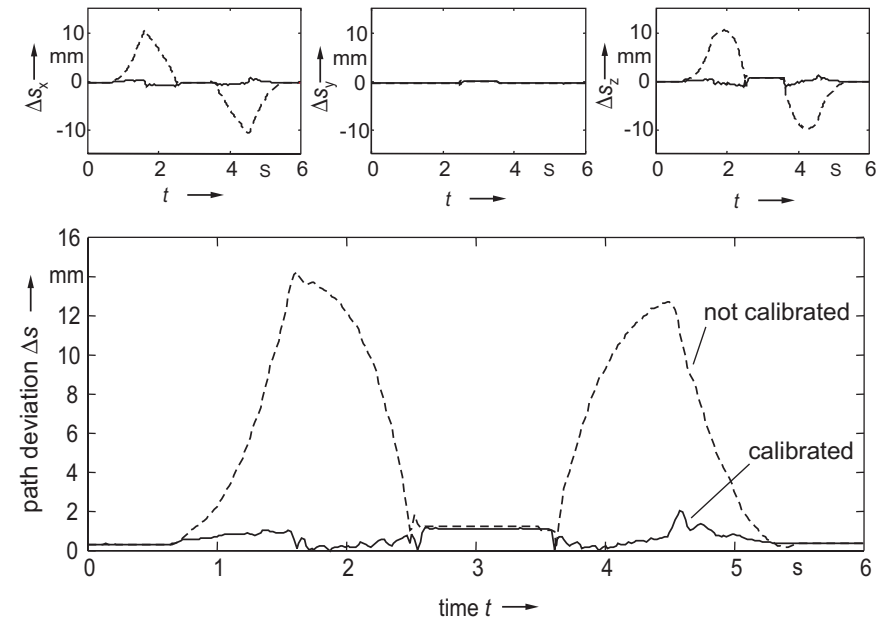

Fig. 6: Measured path deviation of the calibrated and of the not calibrated robot

ation of the end effector is of approx. $14 \mathrm{~mm}$ in the case 
of the not calibrated robot and reduces to approx. $2 \mathrm{~mm}$ for the calibrated robot.

The remaining path errors of the calibrated robot are due to the following reasons that will be taken into account in future:

- Joint 2 of the robot under test shows a drive backlash and an error that depends on the direction of the movement.

- The paths measured for the identification of the parameters differ in the boundary conditions from the path tested here (especially as joint angle velocity and joint position are concerned).

\section{Summary and discussion}

With this dynamic calibration procedure an improvement of the path accuracy of industrial robots, which gains more and more importance, up to $85 \%$ can be achieved. The procedure has been developed to be relatively independent from the type of robot to be calibrated. The method is applicable without knowledge of the structure of the joint controller or of the mass moments of inertia. Only the robot's geometry must be given and the robot needs to have an interface that allows the manipulation of the desired joint values.

\section{Acknowledgement}

The authors are grateful to the Deutsche Forschungsgemeinschaft (DFG) for its support.

\section{References}

[1] S. Spiess, M. Vincze, and M. Ayromlou, "On the calibration of a $6 \mathrm{~d}$ laser tracking system for contactless, dynamic robot measurements", in Proceedings of the IEEE Instrumentation $\&$ Measurement Technology Conference, IMTC, Part, (Ottawa), pp. 12031208, (1997).

[2] G. D. v. Albada and A. Lagerberg, J. M. Visser, "A low-cost pose-measuring system for robot calibration", Tech. Rep., University of Amsterdam, Faculty of Mathematics and Computer Science, (1996).

[3] E. v. Hinüber, Bahn- und Positionsvermessung von Industrierobotern mit inertialen Meßsystemen. $\mathrm{PhD}$ thesis, Universität des Saarlandes, (1993).

[4] K. Schröer, S. L. Albright, and M. Grethlein, "Complete, minimal and model-continuous kinematic models for robot calibration", Robotics and ComputerIntegrated Manufacturing, vol. 13, no. 1, pp. 73-85, (1997).
[5] E. Roos, A. Behrens, and S. Anton, "RDS - realistic dynamic simulation of robots", in 28th International Symposium on Robotics, (Detroit, USA), (1997).

[6] B. Diewald, Über-alles-Kalibrierung von Industrierobotern zur lokalen Minimierung der Posefehler. $\mathrm{PhD}$ thesis, Universität des Saarlandes, (1995).

[7] ISO, ISO 9283, Manipulating Industrial Robots Performance Criteria and Related Test Methods. International Standardization Organisation, (1993).

[8] F. Lange and G. Hirzinger, "Learning of a controller for non-recurring fast movements", Special Issue on Behavior and Learning of the Journal Advanced Robotics, p. 17, (1996).

[9] J. Karner and H. Janocha, "Hybrid controller for adaptive link control of industrial robots", Journal of Intelligent and Robotic Systems, vol. 20, no. 2, pp. 93-104, (1997).

[10] E. Roos, Anwendungsorientierte Meß- und Berechnungsverfahren zur Kalibrierung off-line programmierter Roboterapplikationen. Fortschritt-Berichte VDI Reihe 8, Nr. 709, Düsseldorf: VDI Verlag, (1998).

[11] G. Pritschow, H. Klingel, and B. Renz, "Knickarmroboter zur 3-D-Laserbearbeitung", wt - Produktion und Management, vol. 85, pp. 318-322, (1995).

[12] "RoboCal, closing the gap between model and reality." Fraunhofer-Institut für Produktionsanlagen und Konstruktionstechnik (IPK), Berlin, (1997).

[13] P. S. Roçadas and R. S. McMaster, "Robot and cell calibration using a contact measurement system", in Proceedings of the 5th UK Mechatronics Forum Inernational Conference, Vol. 2, (Portugal), pp. 399-404, (1996).

[14] A. Visser, E. Kohlhaas, and K. Steffen, "Vereinfachtes Kalibrieren von Industrierobotern", Werkstattstechnik, vol. 88, no. $1 / 2$, pp. 49-52, (1998). 